\title{
Learning-induced synaptic diversity and quantification of intra-hippocampal entropy
}

\author{
Volume 8 Issue I - 2017

\section{Dai Mitsushima} \\ Department of Physiology, Graduate School of Medicine, \\ Yamaguchi University, Japan
}

Correspondence: Dai Mitsushima, Graduate School of Medicine, Yamaguchi University, I - I- I Minami-kogushi, Ube 7558505 Japan, Tel 81-836-22-2210, Fax 81-836-85-3726, Email mitsu@yamaguchi-u.ac.jp

Received: June 22, 2017 | Published: July 24, 2017 ation, ${ }^{2}$ temporal information, ${ }^{3}$ and emotional state ${ }^{4}$ within specific episodes. ${ }^{5}$ However, the basic rules such as how to sustain a piece of specific memory and what associates the memory fragment each other are completely unknown.

Stressful experience sometimes forms strong episodic memory in the hippocampus, which is useful to avoid similar risk in future. As a learning paradigm, we used an inhibitory avoidance (IA) task. In the task, we placed a rat in an illuminated box. When the rat entered into the dark side of box, we applied brief electrical foot shock in the dark side. Even one-time foot shock experience, the rat quickly learns the experienced episode. The rats were returned to the home cage, then 30 min after, we placed the rat in the illuminated box again. We measured the latency in the illuminated box as a learning performance (Figure 1). After the training, we made acute brain slices to analyze synaptic function using slice patch-clamp technique.

Although some aspect of the training-induced synaptic plasticity ${ }^{6}$ is similar to the tetanus stimuli-induced LTP, ${ }^{7}$ we found many novel aspects of plasticity in trained animals. For example, to analyze the plasticity, we recorded the postsynaptic response induced by single vesicle of GABA (mIPSC) or glutamate (mEPSC) in the presence of a $\mathrm{Na}^{+}$channel blocker (Figure 2A). The means of mEPSC amplitude and mIPSC amplitude were calculated in each neuron, and the data was plotted in Figure 2B. Although the amplitudes were low and showed a narrow distribution range in untrained, unpaired, or walk-through rats, IA training not only strengthened AMPA receptor-mediated excitatory inputs, but also increased $\mathrm{GABA}_{\mathrm{A}}$ receptor-mediated inhibitory synaptic inputs in CA1 neurons (Figure 2). These results suggest that the training promoted the diversity of excitatory and inhibitory inputs in CA1 neurons. IA training enhanced the diversity of postsynaptic excitatory/inhibitory responses in CA1 neurons. Question arises as to whether the learning requires the synaptic plasticity. We found bilateral CA1 expression of GluA1-c-tail or MPR-DD, an AMPA receptor-delivery blocker, successfully impaired the learning, ${ }^{9}$ suggesting the contextual learning requires the synaptic delivery of GluA1 containing AMPA receptors. Moreover, recent chromophoreassisted light inactivation technique (CALI) further demonstrated that optical inactivation of synaptic AMPA receptors can erase acquired memory. ${ }^{10}$ These results further showed that newly delivered GluA1containing AMPA receptors contribute to forming contextual memory.

As to the inhibitory synaptic plasticity, nicotinic a7 receptor antagonist is known to block the strengthening of GABAA receptormediated synapses. ${ }^{8,11}$ Since pharmacological blockade of the plasticity in bilateral CA1 impaired the learning, contextual learning seems to require the inhibitory synaptic plasticity in the CA1. Moreover,

optogenetic enhancement and suppression technique further revealed the role of the GABAergic inhibitory synapses in learning. ${ }^{12}$ Taken together, I hypothesized that the contextual learning requires the training-induced synaptic plasticity at both excitatory and inhibitory synapses in CA1 (Figure 3).

The learning-induced synaptic diversity can be analyzed mathematically. By calculating the appearance probability of each point of Figure 2B, data from each neuron can be converted to self-entropy (bit) using the information theory of Ralph Hartley ${ }^{19}$ and Claude E Shannon. ${ }^{13}$ A point with high appearance probability (around the mean level) indicates low self-entropy, while a point with very rare probability (a deviated point) indicates high self-entropy. Compared with untrained rats, the self-entropy per neuron was clearly increased in IA-trained rats but not in unpaired or walk-through rats. ${ }^{8}$ This analysis suggests a learning-induced increase in the amount of information in CA1 neurons. Although each neuron showed different self-entropy level, we calculated self-entropy level per single CA1 neuron after the training. ${ }^{14}$ By multiplying $4.0 \times 105$, the total number of CA1 neurons in rats, ${ }^{15}$ we could estimate a possible amount of intra-CA1 self-entropy after the training.

Synaptic inputs regulate neuron firing according to the all-or-none principle. ${ }^{16}$ As neurons are considered an all-or-none device,,${ }^{17,18}$ one neuron can handle 1-bit of memory per clock cycle ( $\log 22=1$ bit) according to a principal formula of information theory. ${ }^{19}$ Based on the principle, computational theory proposed a role of the hippocampus as a kind of memory device. ${ }^{20}$ Our previous finding of a logarithmic correlation between the number of cells blocking plasticity and learning performance ${ }^{9}$ may provide evidence that each CA1 neuron transmits binary data, forming a contextual memory, such as what, where, or when.

Since many diverse features contain entropy, the synapse is not a solo factor to be analyzed. For example, $3.1 \times 10^{9}$ base pairs $^{21}$ of the human genome quaternary system ${ }^{22,23}$ are identical to $6.2 \mathrm{x}$ $10^{9}$ bits in a binary system. However, $6.2 \times 10^{9}$ bits contain only 755 
megabytes ( 1 byte $=8$ bits $)$, the amount is not more than one hour of short movies. Although postnatal maternal care affect the offspring epigenetic programming, ${ }^{24}$ postnatal experience cannot affect the genomic quaternary information. Because our brains process much more information per day, an understanding of genome information cannot be sufficient to reveal the learning systems.

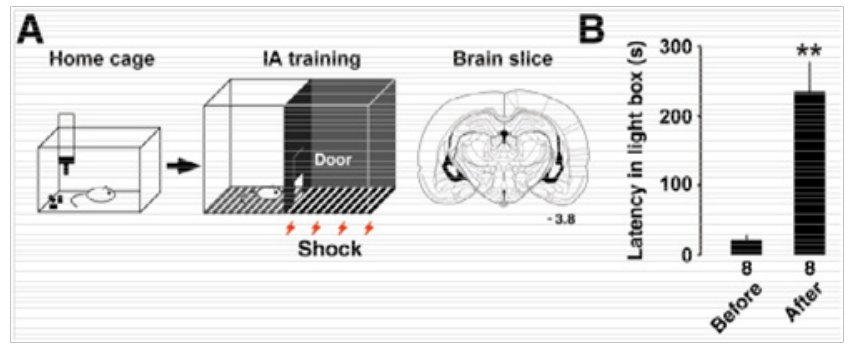

Figure IA Schema of inhibitory avoidance (IA) task. Rats were experienced an episode with an electrical foot shock. B: After the experience, the rats showed longer latency in the illuminated side of the box. ${ }^{8}$

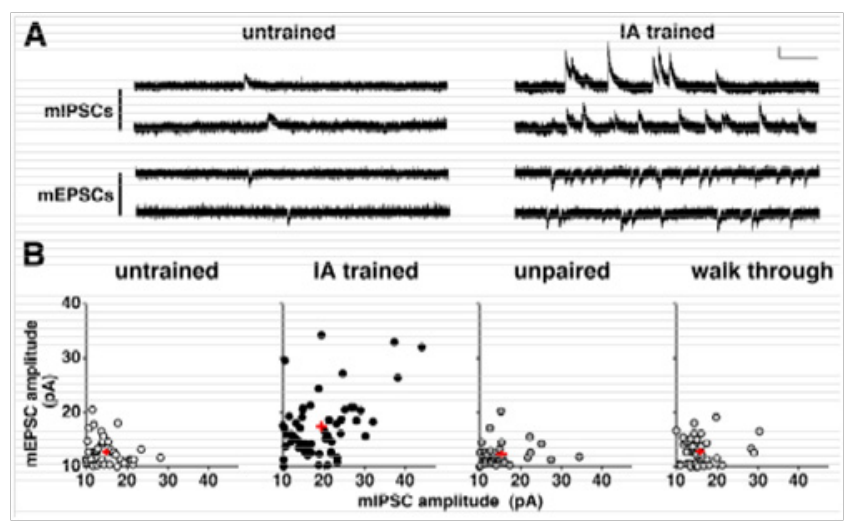

Figure 2A \& 2B Examples of miniature postsynaptic response induced by I synaptic vesicle of glutamate ( $\mathrm{mEPSC}$ ) or GABA (mIPSC). Vertical bar $=20 \mathrm{pA}$ horizontal bar $=200 \mathrm{~ms}$. B: The amplitude of mEPSCs or mIPSCs was averaged and plotted. One plot represents the data from a single CAI neuron.

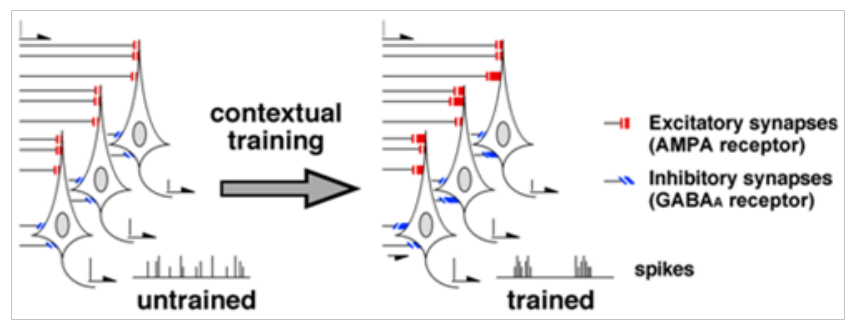

Figure 3 Current hypothesis of learning \& memory. Contextual training induces diversity of the synaptic inputs in CAI neurons. Since GABAergic inputs are critical to synchronize the spike firing, ${ }^{29}$ the multiple CAI neurons probably form diversified ripple-like events after training. Interestingly, all ripples or ripple-like events in many laboratoly groups show different shape. ${ }^{25-28}$

The diversified excitatory/inhibitory synapses after training may create the diversity of ripple-like on/off firing in hippocampal CA1 (Figure 3). In fact, hippocampal ripple-like events seem to exhibit diverse features ${ }^{25,26}$ and phase-locked $\left(\approx 180^{\circ}\right)$ with theta waves. ${ }^{27}$ Interestingly, all the published ripple-like events from many research groups were not identically same. The ripple-like firing may code experienced information, as selective suppression of hippocampal ripples impairs spatial memory in dorsal CA1. ${ }^{28}$ Moreover, we previously found that contextual learning requires the plasticity at excitatory/inhibitory synapses in CA1.$^{8,9}$ Our results, together with the previous studies, we hypothesized that the excitatory/inhibitory synaptic plasticity creates the ripple-like events to process the experienced context by using the theta phase-locked processing system. We are going to analyze the diversity of the ripple-like events in the CA1 in trained animals, that would be necessary to decode intra-CA1 information. ${ }^{29}$

\section{Acknowledgments}

None.

\section{Conflicts of interest}

Author declares there are no conflicts of interest.

\section{Funding}

None.

\section{References}

1. Scoville WB, Milner B. Loss of recent memory after bilateral hippocampal lesions. J NeurolNeurosurgPsychiatry. 1957;20(1):11-21.

2. Wills TJ, Cacucci F, Burgess N, et al. Development of the hippocampal cognitive map in preweanling rats. Science. 2010;328(5985):1573-1576.

3. Mitsushima D, Takase K, Funabashi T, et al. Gonadal steroids maintain 24-h acetylcholine release in the hippocampus: organizational and activational effects in behaving rats. J Neurosci. 2009;29(12):3808-3815.

4. Chen G, Wang LP, Tsien JZ. Neural population-level memory traces in the mouse hippocampus. PLOS ONE. 2009;4(12):e8256.

5. Gelbard-Sagiv H, Mukamel R, Harel M, et al. Internally generated reactivation of single neurons in human hippocampus during free recall. Science. 2008;322(5898):96-101.

6. Whitlock JR, Heynen AJ, Shuler MG, et al. Learning induces long-term potentiation in the hippocampus. Science. 2006;313(5790):1093-1097.

7. Hayashi Y, Shi SH, Esteban JA, et al. Driving AMPA receptors into synapses by LTP and CaMKII: requirement for GluR1 and PDZ domain interaction. Science. 2000;287(5461):2262-2267.

8. Mitsushima D, Sano A, Takahashi T. A cholinergic trigger drives learninginduced plasticity at hippocampal synapses. Nature Communications. 2013;4:2760.

9. Mitsushima D, Ishihara K, Sano A, et al. Contextual learning requires synaptic AMPA receptor delivery in the hippocampus. Proc Natl Acad Sci USA. 2011;108(30):12503-12508.

10. Takemoto $\mathrm{K}$ et al. Optical inactivation of synaptic AMPA receptors erases fear memory. Nat Biotechnol. 2016;35(1):38-47.

11. Townsend, Whyment A, Walczak JS, et al. $\alpha 7$-nAChR agonist enhances neural plasticity in the hippocampus via a GABAergic circuit. $J$ Neurophysiol. 2016;116(6):2663-2675.

12. Chen SX, Kim AN, Peters AJ, et al. Subtype-specific plasticity of inhibitory circuits in motor cortex during motor learning. Nat Neurosci. 2015;18(8):1109-1115.

13. Shannon CE. A mathematical theory of communication. Bell System Technical Journal. 1948;27:379-423, 623-656.

14. Ono K, Mitsushima D. Learning creates diversity of excitatory and inhibitory synapses in the hippocampal CA1: a possible amount of information at a single synapse. J Physiol Sci. 2017;67 Suppl 1.

15. Andersen P, Morris R, Amaral D, et al. The Hippocampus Book. (1st edn), Oxford University Press, USA. 2006. p.1-872.

16. Kandel ER, Schwartz JH, Jessell TM, et al. Principles of Neural Science. (5th edn), the McGraw-Hill Companies, Inc, New York, USA. 2013. p. $1-1760$. 
17. Cannon WB. Biographical Memoir: Henry Pickering Bowdich. National Academy of Sciences, Washington, USA. 1924. p.181-196.

18. Wiener N. Cybernetics: or control and communication in the animal and machine. (2nd edn), MIT press, Cambridge, UK. 1961. p.1-232.

19. Hartley RVL. Transmission of Information. Bell System Technical Journal. 1928;7(3):535-563.

20. Marr D. Simple memory: a theory for archicortex. Philos Trans R Soc B Biol Sci. 1977;262(841):23-81.

21. International Human Genome Sequencing Consortium. Finishing the euchromatic sequence of the human genome. Nature. 2004;431(7011):931-945

22. Nirenberg M, Leder P. RNA codewords and protein synthesis. Science. 1964;1453639):1399-1407.

23. Watson JD, Crick FHC. Genetical implications of the structure of deoxyribonucleic acid. Nature. 1953;171:964-967.
24. Weaver ICG, et al. Epigenetic programming by maternal behavior. Nature Neuroscience. 2004;7:847-854.

25. O'Keefe J, Nadel L. The Hippocampus as a Cognitive Map. Oxford University Press, USA. 1978. P.1-296.

26. Ishikawa J, Mitsushima D. Real-time change of neural activity in hippocampal CA1 after the experienced episodes: restraint stress and first encounters with female, male, and object. Soc Neurosci Abstr, USA. 2016.

27. Csicsvari J, Hirase H, Czurkó A, et al. Oscillatory coupling of hippocampal pyramidal cells and interneurons in the behaving rat. $J$ Neurosci. 1999;19(1):274-287.

28. Girardeau G, Benchenane K, Wiener SI, et al. Selective suppression of hippocampal ripples impairs spatial memory. Nat Neurosci. 2009; $12: 1222-1223$.

29. Fisahn A, Pike FG, Buhl EH, et al. Cholinergic induction of network oscillations at $40 \mathrm{~Hz}$ in the hippocampus in vitro. Nature. 1998;394(6689):186-189. 